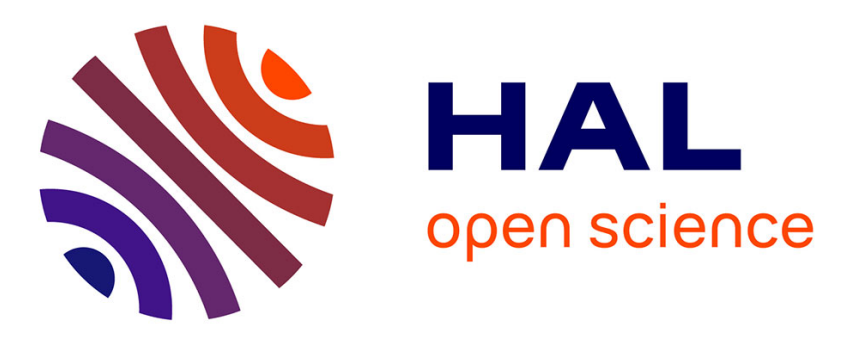

\title{
Dosage radioimmunologique des gonadotropines plasmatiques chez Carassius auratus, au cours du nycthémère et pendant l'ovulation
}

\author{
Bernard Breton, Roland Billard, Bernard Jalabert, G. Kann
}

\section{To cite this version:}

Bernard Breton, Roland Billard, Bernard Jalabert, G. Kann. Dosage radioimmunologique des gonadotropines plasmatiques chez Carassius auratus, au cours du nycthémère et pendant l'ovulation. General and Comparative Endocrinology, 1972, 18, pp.463-468. 10.1016/0016-6480(72)90027-5 . hal01605395

\section{HAL Id: hal-01605395 \\ https://hal.science/hal-01605395}

Submitted on 2 Jun 2020

HAL is a multi-disciplinary open access archive for the deposit and dissemination of scientific research documents, whether they are published or not. The documents may come from teaching and research institutions in France or abroad, or from public or private research centers.
L'archive ouverte pluridisciplinaire $\mathbf{H A L}$, est destinée au dépôt et à la diffusion de documents scientifiques de niveau recherche, publiés ou non, émanant des établissements d'enseignement et de recherche français ou étrangers, des laboratoires publics ou privés. 


\title{
Dosage radioimmunologique des gonadotropines plasmatiques chez Carassius auratus, au cours du nycthémère et pendant l'ovulation
}

\author{
B. BRETON, R. BILLARD, B. JALABERT, eT G. KANN ${ }^{1}$ \\ Institut National de la Recherche Agronomique, Station Centrale de \\ Physiologie animale C.N.R.Z. 78-Jouy-en-Josas, France
}

Received April 8, 1971

\begin{abstract}
L'évolution du taux de gonadotropines a été suivie dans le plasma par dosage radioimmunologique chez Carassius auratus au cours du nycthémère et pendant la période d'ovulation. Les résultats mettent en évidence :

1. L'existence d'une forte décharge hormonale dans le sang au moment de l'ovulation (Fig. 5).

2. Une évolution cyclique du contenu hormonal plasmatique au cours du nycthémère particulièrement nette chez les femelles (Fig. 3), avec un maximum à 11 heures du matin dans le nycthémère de juillet.

Changes in plasmatic gonadotropin level were observed by radioimmunologic assay in Carassius auratus for a $24-\mathrm{hr}$ period during ovulation. The following results were found:

1. A high hormone discharge into the blood at the time of ovulation (Fig. 5).

2. A particularly distinct change of plasmatic hormone content over a 24 -hr period in the females (Fig. 3) which is at its maximum at $11 \mathrm{AM}$ in the 24 -hr period of July.
\end{abstract}

L'hypothèse d'une décharge d'hormone gonadotrope hypophysaire dans le sang des Poissons au moment de la fraie a été émise par Matthews (1938) chez Fundulus et Vivien (1941) chez Gobius paganellus. Un certain nombre de travaux ultérieurs tendent à confirmer l'existence d'une telle décharge :

La cytologie hypophysaire révèle la présence de granulations dans les cellules supposées gonadotropes de l'hypophyse au cours de la maturation des gonades et une dégranulation considérée comme une décharge au moment de la fraie. Les observations ont été faites principalement en microscopie photonique (Pickford et Atz, 1957 ; Ramaswami, 1962 ; Ball, 1965 ; Hoar, 1970 ; Barr, 1963a,b,c ; Olivereau, 1967), mais également en microscopie électronique (Leatherland, 1970).

${ }^{1}$ G. Kann, Station de Physiologie de la Lactation, C.N.R.Z. 78- Jouy-en-Josas.

(C) 1972 by Academic Press, Inc.
L'activité biologique du contenu gonadotrope hypophysaire, mesurée au cours du cycle reproducteur, est maximum pendant la période de développement des gonades et minimum après la fraie (Gerbilsky, 1940 ; Barr et Hobson, 1964 ; Swift et Pickford, 1965 ; Clemèns et Johnson, 1965 ; Singh, 1970). Cependant les dosages biologiques ne sont pas assez sensibles pour permettre d'apprécier la quantité d'hormone circulante. La mise au point d'une technique de dosage radioimmunologique des gonadotropines plasmatiques chez la Carpe et le Cyprin a permis dans cette étude préliminaire, l'étude de l'évolution du taux d'hormone circulante au cours du cycle circadien et de l'ovulation.

\section{MATERIEL ET METHODES}

Les expériences conduites en juillet 1970 sur des animaux d'une souche élevée au laboratoire ont porté sur des mâles et des femelles placés 
séparément par groupe de 5 dans des bacs de 100 litres et soumis aux conditions naturelles de lumière et de température. Le sang (200 à 300 ul) prélevé par ponction cardiaque (adaptation chez le Cyprin de la technique rutilisée chez la Truite par Dorson, 1968) est recueilli sur EDTA. Le plasma obtenu après centrifugation de 20 minutes à $6000 \mathrm{~T} / \mathrm{Mn}$ à $4^{\circ} \mathrm{C}$ est stocké à $-35^{\circ} \mathrm{C}$ et n'est décongelé qu'au moment du dosage (décembre 1970).

Les lots d'animaux correspondent chacun à une tranche horaire de prélèvement journalier ; ainsi, chaque animal n'est prélevé qu'une fois par jour. Pour les femelles, il y a 10 prélèvements par jour et par conséquent 10 lots ; il n'y a que 5 prélèvements chez les mâles, soit 5 lots.

L'ovulation, vérifiée a posteriori par l'émission abondante d'oeufs mûrs après une légère pression abdominale, est contrôlée lors de chaque prélèvement.

L'hormone gonadotrope de Carpe c-HG (Burzawa-Gérard, 1971), active sur la restauration de la spermatogenèse du Guppy (Billard et al., 1970), est marquée par ${ }^{131} \mathrm{I}$ ( $\mathrm{IS}_{\mathbf{3}} \mathrm{CEA}$, France) selon la méthode de Greenwood et al. (1963). L'hormone radioiodée est séparée des iodures libres sur Sephadex G-50 et purifiée ensuite sur Sephadex G-100. Un rendement moyen d'iodation de $90 \%$ permet d'obtenir une radioactivité spécifique de 180 à $200 \mu \mathrm{Ci} / \mu \mathrm{g}$ de $\mathrm{c}-\mathrm{HG}$.

La technique de dosage utilisée a été mise au point initialement sur des extraits hypophysaires de Carpe (Breton et al., 1971) avec une sensibilité de $0.625 \mathrm{ng} / \mathrm{ml}$. Après avoir préalablement testé la spécificité du système pour l'hormone gonadotrope de Cyprin, la technique de dosage a été adaptée au dosage de l'hormone plasmatique de cette même espèce.

\section{RESULTATS}

\section{Spécificité du système vis-à-vis de l'hormone gonadotrope de Cyprin}

Les courbes standards de dosages réalisées avec l'hormone purifiée (c-HG) et des extraits hypophysaires de Carpe et de Cyprin (Fig. 1) montrent qu'aux mêmes

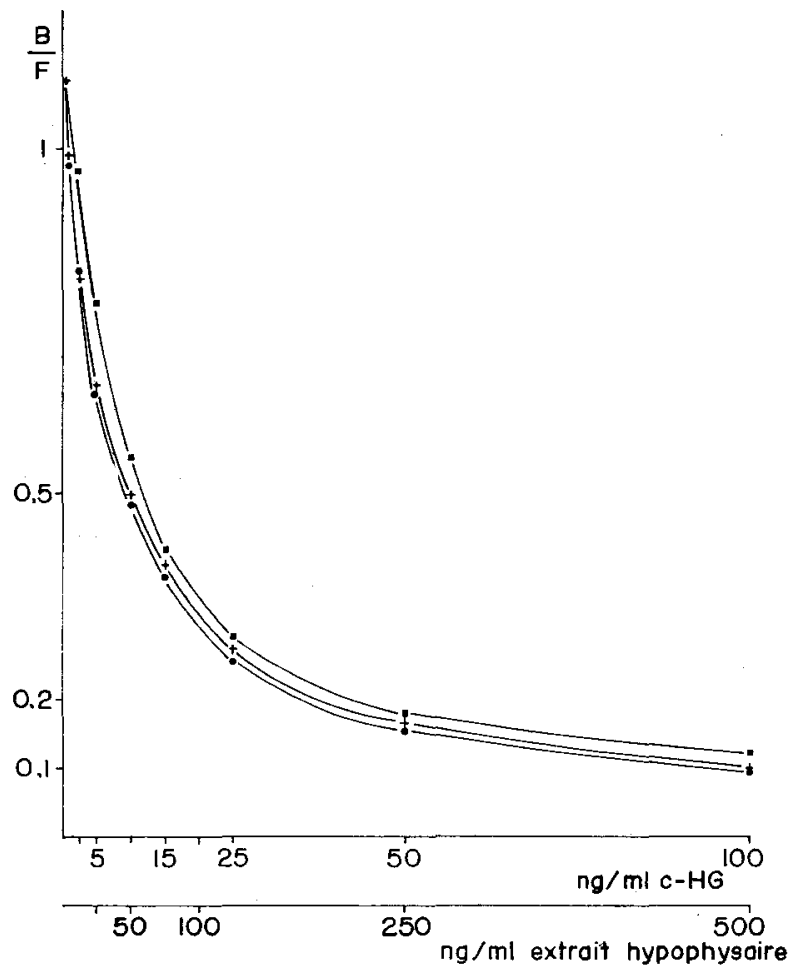

FIG. 1. Courbes standards de dosage obtenues avec: (1) l'hormone gonadotrope de Carpe c-HG $(+-++)$, (2) l'extrait hypophysaire de Carpe (

dans le système immunologique $\mathrm{c}-\mathrm{HG}$-Anti $\mathrm{c}-\mathrm{HG}$. 
concentrations, les courbes de décroissance $\mathrm{B} / \mathrm{F}$ (hormone liée/hormone libre) sont identiques et parallèles à la courbe c-HG de référence. Le système radioimmunologique autorise done le dosage de l'hormone gonadotrope du Cyprin.

\section{Dosage plasmatique (Fig. 2)}

La courbe standard de référence établie avec c-HG n'est pas modifiée par l'addition de $10 \mu \mathrm{l}$ de sérum de Cyprin hypophysectomisé dans chaque échantillon. Une courbe réalisée en remplaçant les échantillons de c-HG par des quantités croissantes de plasma de Cyprin normal se superpose à la courbe de dosage standard de référence établie avec c-HG. La décroissance de B/F n'est donc due qu'à l'hormone circulante. La sensibilité obtenue est de $0.625 \mathrm{ng} / \mathrm{ml}$ comme pour des extraits hypophysaires.

\section{Evolution du taux d'hormone plasmatique chez la femelle}

Existence d'un cycle circadien. L'ensemble des résultats obtenus sur une période de 6 jours pour des femelles n'ayant pas ovulé est regroupé par tranches horaires sur un seul nycthémère moyen correspondant à la période étudiée (soit 15 à 16 heures d'éclairement par jour) (Fig. 3). Le taux d'hormone circulante qui est de $5.75 \pm$ $1.35 \mathrm{ng} / \mathrm{ml}$ de plasma au début de la journée augmente significativement à 11 heures $(9.55 \pm 1.18 \mathrm{ng} / \mathrm{ml})$ et diminue ensuite de façon significative lors du prélèvement suivant. Il subit ensuite des fluctuations non significatives caractérisées par l'existence de 2 pics à 15 et à 18 heures. En fin de journée le taux d'hormone est plus faible qu'au début et atteint $3.34 \pm$ $0.98 \mathrm{ng} / \mathrm{ml}$.

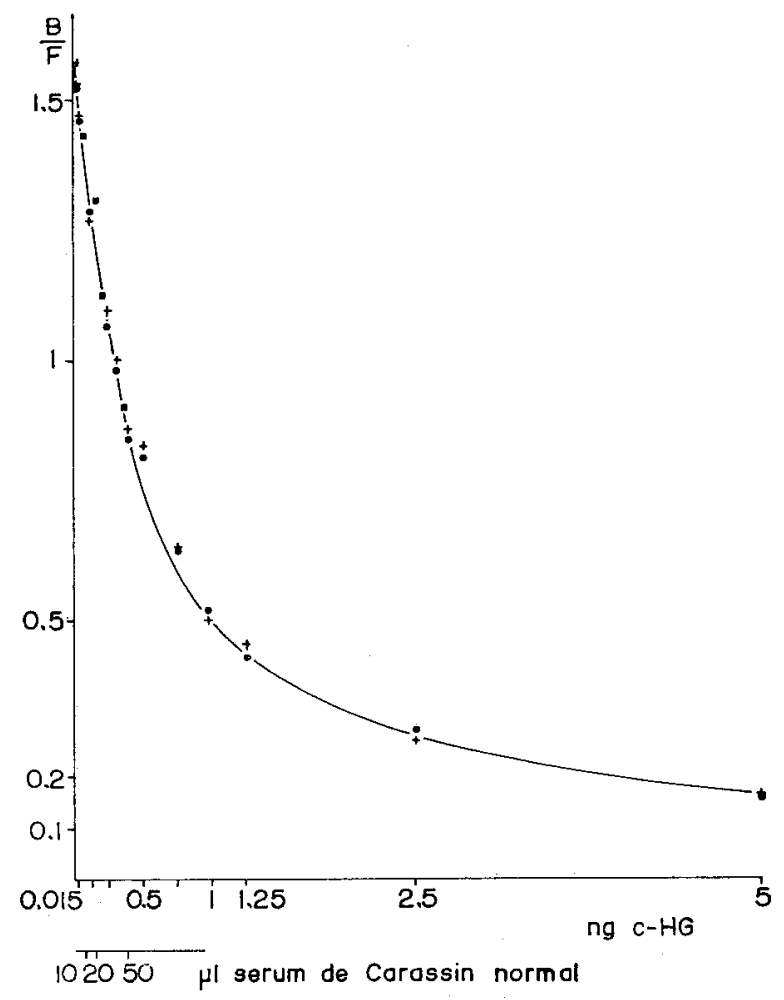

FIG. 2. Effet de l'addition de plasma de Cyprin sur la courbe standard de dosage. Courbe normale $+\ldots+$; effet de l'addition de $10 \mu \mathrm{l}$ de sérum de Cyprin hypophysectomisé dans chaque échantillon, ; effet de l'addition de quantité croissante de sérum de Cyprin normal, 


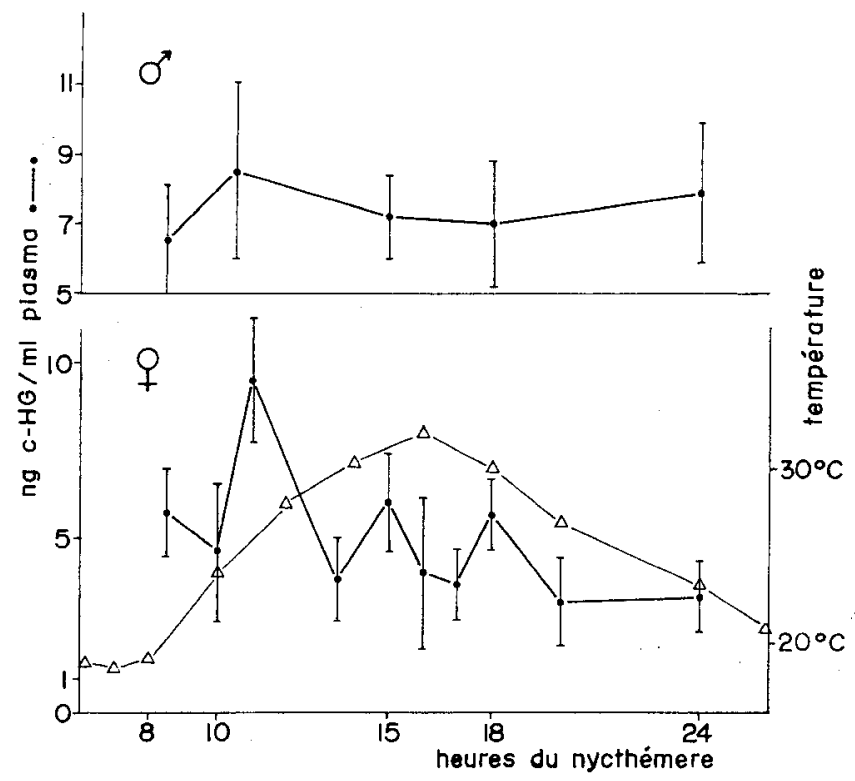

Frg. 3. Variations nycthémérales du taux d'hormone gonadotrope plasmatique chez le Cyprin mâle et femelle. Les valeurs portées représentent les moyennes sur 6 jours consécutifs (intervalle de confiance, $P=$ 0.05). Taux d'hormone circulante $(\mathrm{ng} / \mathrm{ml}) ; \triangle \longrightarrow \Delta$, variations thermiques au cours $\mathrm{du}$ nycthémère.

Le calcul du taux moyen journalier d'hormone circulante fait apparaître des variations au cours de la période de prélèvement de 6 jours (Fig. 4). Les variations sont en relation étroite avec l'amplitude des écarts de température (différence entre maximum et minimum enregistrés dans la journée).

Existence d'un cycle ovulatoire. Les résultats obtenus sur 14 femelles sont regroupés sur la Fig. 5 et font apparaître une importante décharge au moment de l'ovulation où le taux d'hormone circulante

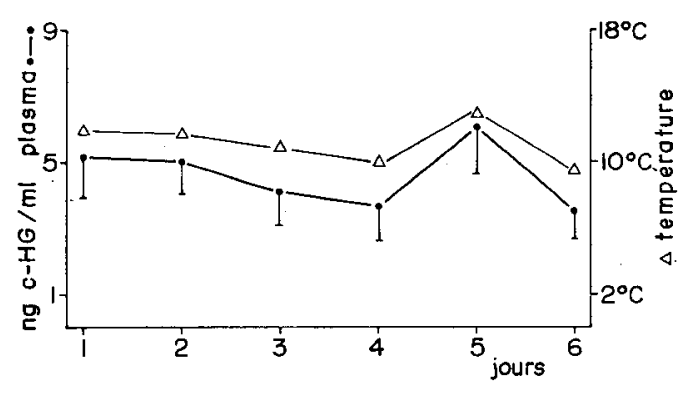

FIG. 4. Variations du taux d'hormone circulante moyen au cours de 6 jours consécutifs (intervalles de confiance, $P=0.05$ ). - Taux d'hormone circulante $(\mathrm{ng} / \mathrm{ml}) ; \triangle \longrightarrow \triangle$, écarts de température.

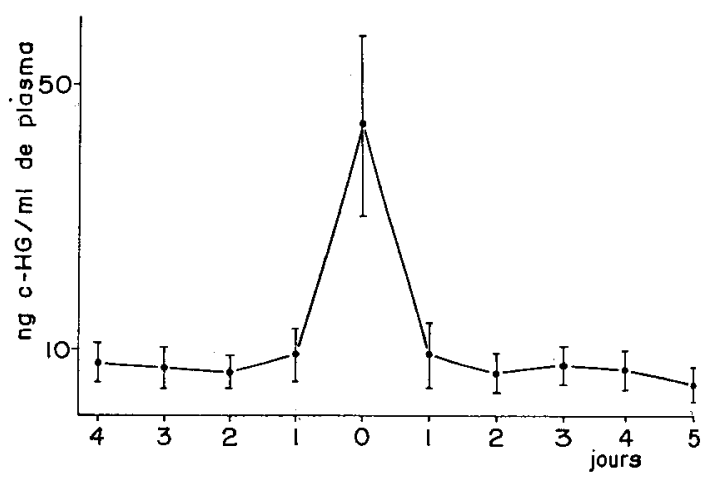

Fig. 5. Evolution du taux d'hormone plasmatique au cours de l'ovulation. Le graphique donne les valeurs moyennes obtenues sur 14 animaux (intervalles de confiance, $P=0.05$ ). Le jour d'ovulation a été pris comme jour $\mathrm{J}_{0}$.

atteint $49.30 \pm 12.92 \mathrm{ng} / \mathrm{ml}$. Dans les conditions de l'expérience un seul pic est décelable et la quantité d'hormone plasmatique est la même avant et après l'ovulation $(6.31 \pm 1.42$ à $9.36 \pm 2.00 \mathrm{ng} / \mathrm{ml})$. Ce "taux moyen de base" est significativement plus élevé que celui des femelles n'ayant pas ovulé pendant cette même période $(2.71 \pm 1.6$ à $6.03 \pm 1.37 \mathrm{ng} / \mathrm{ml})$. 
L'ovulation intervient généralement après une séquence de 3 ou 4 jours chauds.

\section{Evolution du taux d'hormone plasmatique chez le mâle}

L'évolution du taux d'hormone circulante au cours du nycthémère est moins marquée que chez la femelle (Fig. 4). Le maximum se situe cepandant à 11 heures, mais les différences ne sont pas significatives. Ces mâles expérimentaux présentaient une légère spermiation (émission de très faibles quantités de sperme après pression abdominale).

\section{DISCUSSION}

\section{Techniques de Dosage}

Le dosage radioimmunologique de l'hormone gonadotrope de Carpe c-HG, déjà mis en oeuvre au niveau hypophysaire (Breton et al., 1971) peut être également adapté au dosage de l'hormone circulante. Chez le Cyprin l'addition de plasma d'animaux hypophysectomisés n'entraîne pas de modification de la courbe standard de dosage. Il n'y a donc pas de précipitations aspécifiques dues à la présence de protéines plasmatiques non gonadotropes.

\section{Cycle Nycthéméral}

Les résultats obtenus mettent en évidence des variations du taux d'hormone gonadotrope circulante au cours du nycthémère. L'évolution parallèle de la quantité moyenne journalière d'hormone circulante et des variations thermiques quotidiennes laisse supposer que le niveau moyen d'hormone plasmatique pourrait être sous la dépendance de la température (Fig. 4). Cependant l'existence d'un pic maximum de décharge qui se renouvelle tous les matins à 11 heures quelles que soient les variations de température et avant le maximum thermique suggère en outre une action de la photopériode.

Une relation entre un haut niveau hormonal et uné température élevée a déjà été supposée chez le Lézard (Licht et Pearson, 1969).

\section{Ovulation}

Il existe au moment de l'ovulation une décharge importante d'hormone dans le sang, mais les conditions de l'expérience (l prélèvement par animal et par jour) ne permettent pas de déterminer avec précision la durée de la décharge. L'apparition de l'ovulation après une séquence de jours chauds laisse également supposer un effet itératif de la température, bien que les effets respectifs de la température et de la photopériode n'aient pas été dissociés dans cette étude. Un niveau hormonal élevé semble donc nécessaire à l'ovulation qui se produit rarement dans des conditions artificielles d'élevage, nécessitant alors dans la pratique une supplémentation gonadotrope exogène qualifiée d'hypophysation.

Ces résultats sont en accord avec les observations cytologiques réalisées au moment de la fraie.

Cette étude descriptive qui met en évidence un cycle nycthéméral dans la teneur hormonale gonadotrope plasmatique et une décharge ovulante importante ne permet pas de dissocier avec certitude les effets de la lumière et de la température dans les conditions naturelles et sera complétée par une analyse expérimentale permettant de dissocier les effets respectifs de ces deux facteurs.

\section{REMERCIEMENTS}

Madame E. Burzawa-Gérard du Muséum d'Histoire Naturelle de Paris nous a fourni l'hormone gonadotrope de Carpe c-HG.

L'analyse statistique a été réalisée par Mademoiselle Aline Solari.

\section{BIBLIOGRAPHIE}

BALL, J. N. (1965). Reproduction in female bony fishes. Symp. Zool. Soc. London 1, 105-135.

BARR, W. A. (1963a). The endocrine control of the sexual cycle in the plaice, Pleuronectes platessa $(L)$. I. Cyclical changes in normal ovary. Gen. Comp. Endocrinol. 3, 197-204.

BARR, W. A. (1963b). The endocrine control of the sexual cycle in the plaice, Pleuronectes platessa $(L$.$) . II. The endocrine control of ovo-$ genesis. Gen. Comp. Endocrinol. 3, 205-215.

BARR, W. A. (1963c). The endocrine control of the sexual cycle in the plaice, Pleuronectes platessa (L.). III. The endocrine control of 
spermatogenesis. Gen. Comp. Endocrinol. 3 216-225.

Barr, W. A. (1965). The endocrine physiology of fishes. Annu. Rev. Oceanogr. Mar. Biol. 3, 257298.

Barr, W. A., et Hobson, B. M. (1964). The endocrine control of the sexual cycle in the plaice, Pleuronectes platessa (L.) IV. Gonadotropic activity of the pituitary gland. Gen. Comp. Endocrinol. 4, 608-613.

BEACH, A. W. (1959). Seasonal changes in the cytology of the ovary and of the pituitary gland of the goldfish. Can. J. Zool. 37, 615-625.

Billard, R., Burzawa-Gerard, E., et Breton, B. (1970). Regénération de la spermatogenèse du Cyprin hypophysectomisé Carassius auratus $L$. par un facteur gonadotrope hautement purifié de Carpe. C. R. Acad. Sci. 271, 1846-1899.

Breton, B., KanN, G., Burzawa-Gérard, E., ET Billard, R. (1971). Dosage radioimmunologique d'une hormone gonadotrope de Carpe. $C . R$. Acad. Sci. 272, 1515-1517.

Burzawa-Gírard, E. (1971). Purification d'une hormone gonadotropehypophysaire de poisson téléostéen, la carpe (Cyprinuscarpio) Biochimie $53,545-552$.

Clemens, H. P., et Johnson, W. W. (1965). Specificity of the gonadal hydratation factor in the pituitary of some freshwater fishes. Copeia 3, 389-398.

Dorson, M. (1968). Une technique simple de ponction cardiaque chez la Truite. Ann. Biol. Anim. Biochim. Biophys. 8, 441-444.

Fontaine, M. (1969). Contrôle endocrinien de la reproduction chez les Poissons Téléostéens. Verh. Int. Verein Limnol. 17, 611-624.

Gerbilsky, N. L. (1940). Seasonal changes in the gonadotrophic potency of the pituitary gland in fishes. Dokl. Akad. Nauk SSSR 28, 571-573.

Greenwood, F., Hunter, W., et Glover, J. (1963). The preparation of ${ }^{131} \mathrm{I}$ labeled human growth hormone of high specific radioactivity. Biochem. J. 89, 114-123.

HoAR, W. S. (1970). Reproduction. In "Fish Physiology" (W. S. Hoar et D. J. Randall, eds.), Vol. III, pp. 1-59. Academic Press, New York.
Knowles, F., ex Vollrath, L. (1966). Cell types in the pituitary of the eel Anguilla anguilla $L$. at different stages in the life cycle. Z. Zellforsch. Mikrosk. Anat. 69, 474-479.

Leatherland, J. F. (1970). Seasonal variation in the structure and ultrastructure of the pituitary gland in the marine form (Trachurus) of the threespine Stickleback, Gasterosteus aculeatus $L$. II. Proximal pars distalis and neurointermediate lobe. $Z$. Zellforsch. Mikrosk. Anat. 104, 318-336.

Licht, P., et Pearson, A. K. (1969). Effects of mammalian gonadotrophins (FSH and $\mathbf{L H}$ ) on the testes of the lizard Anolis carolinensis. Gen. Comp. Endocrinol. 13, 367-381.

Matrhews, S. A. (1938). The seasonal cycle in the gonads of Fundulus. Biol. Bull. 75, 66 .

Olivereau, M. (1967). Observations sur l'hypophyse de l'Anguille femelle, en particulier lors de la maturation sexuelle $Z$. Zeitforsch. Mikrosk. Anat. 80, 286-306.

Pickford, G. E., et Atz, J. W. (1957). The Physiology of the Pituitary Gland of Fishes," pp. 178-269. Academic Press, New. York.

Ramaswami, L. S. (1962). Endocrinology of reproduction in fish and frog. Gen. Comp. Endocrinol., Suppl. 1, 286-299.

Swift, D. R., En Pickford, G. E. (1965). Seasonal variations in the hormone content of the pituitary gland of the perch Perca fluvialis L. Gen. Comp. Endocrinol. 5, 354-365.

Singh, T. P. (1970). Seasonal variations in the cyanophils and gonadotrophic potency of pituitary gland in relation with the gonadal activity in the catfish Mystus vittatus Bloch. Endokrinologie 56, 292-303.

SundararaJ, B. I. (1960). A study on the correlation between the structure of the pituitary and the changes in the testes of the Indian catfish Heteropneustes. Acta Anat. 40, 305-322.

Vivien, J. H. (1941). Contribution à l'étude de la physiologie hypophysaire dans ses relations avec l'appareil génital, la thyroïde et les corps suprarénaux chez les Poissons Sélaciens et Téléostéens. Bull. Biol. Fr. Belg. 75, 257-309. 